\title{
El mini computador de papy como instrumento de enseñanza de las operaciones básicas para alumnos con limitación visual ${ }^{1}$
}

\author{
Papy's minicomputer as a teaching tool for the basic \\ operations for visually impaired students
}

Minicomputador de Papy como uma ferramenta de

ensino para as operações básicas para os alunos com deficiência visual

Recibido: mayo de 2013

Aceptado: agosto de 2013
Diana Carolina Rubiano ${ }^{2}$

Francy Viviana Zolaque ${ }^{3}$

Wilderson David mantilla Vaca ${ }^{4}$

Christian Andrés Rodríguez Ramírez ${ }^{5}$

\section{Resumen}

La formación en la LEBEM se preocupa por suplir las necesidades de enseñanza-aprendizaje de los estudiantes, brindando espacios de formación que permiten conocer situaciones que un maestro enfrenta en su labor, como la enseñanza a estudiantes con limitación visual. Es así, como surge la propuesta de implementar un material didáctico útil y beneficioso, que permita desarrollar la actividad matemática; un material didáctico adaptado que acompañado del valor pedagógico del juego, facilita captar la atención de los alumnos por las matemáticas, para esto se pretende trabajar y adecuar "El minicomputador de Papy". En este trabajo se presenta una propuesta inclusiva como una nueva alternativa de enseñanza, ya que este material adaptado es útil para niños con y sin limitación visual.

Palabras clave: Aula; recursos didácticos; recursos informáticos; computadores; minicomputador de Papy; alumno; necesidades especiales; alumnos discapacitados; limitación visual; aprendizaje; operaciones matemáticas; material adaptado.

\footnotetext{
Abstract

Training in LEBEM cares about meeting the needs of teaching and student learning, providing opportunities for training that provide insight into situations that a teacher faces in his work, such as teaching students with
}

1 Artículo de Investigación

2 Universidad Distrital Francisco José de Caldas. Bogotá, Colombia. Contacto: nicolcarolina23@hotmail.com

3 Universidad Distrital Francisco José de Caldas. Bogotá, Colombia. Contacto: francy280907@hotmail.com

4 Universidad Distrital Francisco José de Caldas. Bogotá, Colombia. Contacto: davidmantilla_123@hotmail.com

5 Universidad Distrital Francisco José de Caldas. Bogotá, Colombia. Contacto: cardenal093@hotmail.com 
visual impairment. Thus, as there is the proposal to implement a useful and beneficial educational material, allowing the development of mathematical activity, an appropriate teaching material that accompanied the educational value of the game, easy to capture the attention of students in mathematics, for this is to work and adapt "Papy's minicomputer". In this paper we present a proposal as a new alternative inclusive education, as this is useful material adapted for children with and without visual impairment.n.

\section{Keywords:}

classroom, teaching resources, computing resources, computers, minicomputer Papy, student, special needs, students with disabilities, visual impairment, learning math; material adapted.

\section{Resumo}

Formação em LEBEM preocupa atender às necessidades de ensino e aprendizagem dos alunos, proporcionando oportunidades de formação que fornecem informações sobre situações que um professor enfrenta em seu trabalho, como ensinar os alunos com deficiência visual. Assim, como não é a proposta de implementar um material didático útil e benéfica, permitindo que o desenvolvimento da atividade matemática, um material didático apropriado que acompanha o valor educativo do jogo, fácil de capturar a atenção dos alunos em matemática, por isso é trabalhar e se adaptar "minicomputador de Papy". Neste artigo apresentamos uma proposta como uma nova alternativa de educação inclusiva, pois este é um material útil adaptados para crianças com e sem deficiência visual.

Palavras-chave: sala de aula, recursos pedagógicos, recursos de computação, computadores, Papy minicomputador, estudante, necessidades especiais, estudantes com deficiência, deficiência visual, matemática aprendizagem; material adaptado.

\section{Contextualización}

Se diseña una secuencia de actividades en torno a la enseñanza de las operaciones básicas para desarrollar en un aula inclusiva, esta propuesta está basada en la adaptación para personas con limitación visual de un juego común en el proceso de enseñanza-aprendizaje en niños de edades tempranas, una vez adaptado el material tema se realiza una secuencia de actividades la cual inicia con la actividad diagnostico para poder identificar las nociones que tienen los estudiantes acerca de la adición y sustracción, además de presentar el material con el que se pretende desarrollar la propuesta, una vez obtenidos los resultados se diseñan las actividades intentando que el estudiante caiga en un conflicto cognitivo y esto propicie el trabajo autónomo cambiando así sus esquemas de conocimiento y ayudando a la evolución del razonamiento matemático y los proceso algorítmicos, puntualmente operando números enteros.

\section{Referentes teórico prácticos básicos}

Para comenzar, el minicomputador de Papy es un instrumento diseñado para el aprendizaje de la organización de los números en base diez y las correspondientes operaciones entre ellos. Su creador fue Frédérique Papy quien fue un matemático belga; este instrumento "combina el sistema decimal y el binario: recibe la información en base diez, la transforma y procesa en base dos y da un resultado en base diez" (Robayna, 1989). En cuanto la descripción del minicomputador de Papy, este cuenta con placas cuadradas las cuales esta divididas en cuatro casillas y 
fichas de diferentes colores. Estas placas son puestas de manera horizontal, en donde sigue la regla del sistema decimal:

Figura 1.

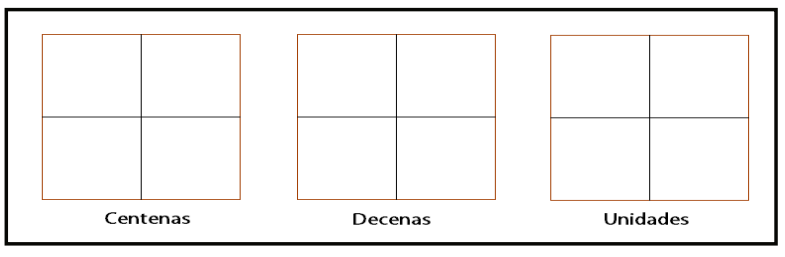

Fuente: Elaboración propia

Esta división de cuatro casillas nos permitirá representar los números del 1 al 9. En honor a Cuisenaire cada tiene el color de las regletas, ubicados de la siguiente manera:

Figura 2.
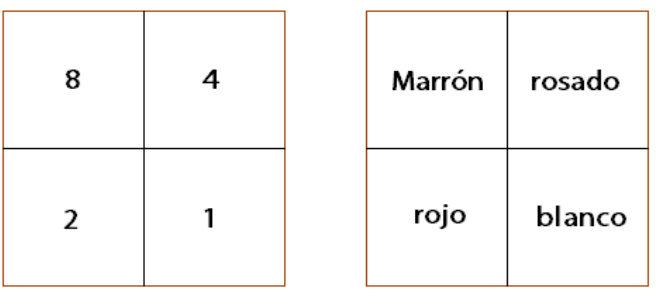

Fuente: Elaboración propia

De esta manera, una ficha ubicada en la casilla blanca representa el numero $1(1=0001)$, una en la roja representa el numero $2(2=0010)$, una en la rosada que es el numero $4(4=0100)$ y una en la marrón que es el numero $8(8=1000)$ y de esta manera números como: $3=0011 ; 5=0101 ; 7=0111$ y $9=1001$.

A continuación presentamos las reglas con las que cuenta el minicomputador de Papy:

- Dos fichas en la casilla blanca equivalen a una en la roja

Figura 3.

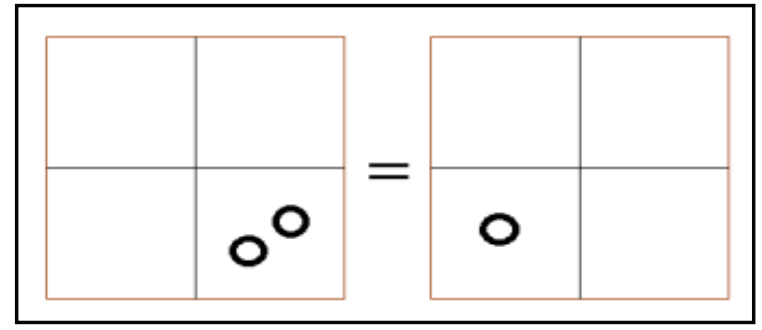

Fuente: Elaboración propia
- Dos fichas en la casilla rija equivalen a una en la rosada

Figura 4.

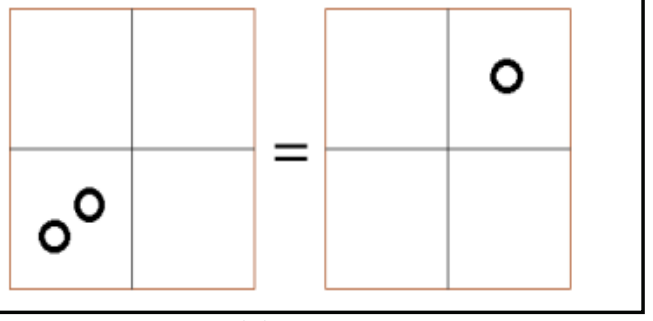

Fuente: Elaboración propia

- Dos fichas en la casilla rosada equivalen a una en la marrón

Figura 5.

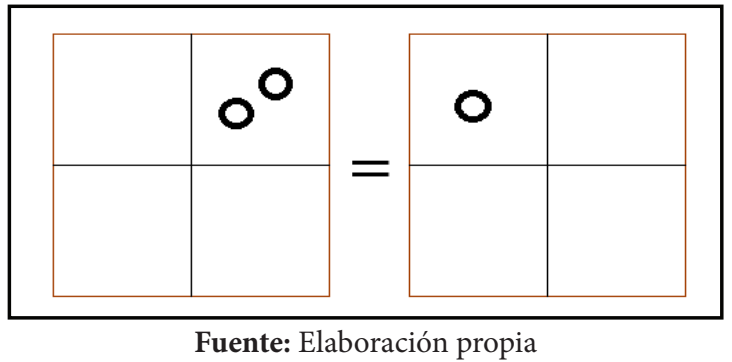

En cuanto a la organización decimal, la regla es "una ficha en la casilla marrón y una en la roja es igual a una ficha en el blanco de la placa situada en la parte izquierda (Robayna, 1989). Este instrumento cuanta con operaciones tales como la adición, sustracción, multiplicación, división, potenciación, radicación y logaritmación.

No obstante, un instrumento que tiene alcances valiosos para el aprendizaje, puede ser una herramienta indispensable para cualquier tipo de estudiante y por tal motivo este debe ser adecuado a las necesidades del mismo, que en nuestro caso es el estudiante con discapacidad visual. En este sentido, siendo un instrumento útil para la actividad matemática, la adaptación debe estar ligada al cumplimiento de algunas destrezas mínimas para que el estudiante pueda afrontar la educación básica (Calderón \& Vega, 2011). De esta manera el desplazamiento, el lenguaje, el desarrollo del sentido del tacto y del sentido del oído son para Calderón y Vega (2011) las áreas fundamentales que se deben reforzar en el proceso de enseñanza-aprendizaje de estos niños. 


\section{Descripción de la actividad}

Siendo una propuesta dirigida a estudiantes con limitación visual a continuación se presentará la adecuación realizada al minicomputador de Papy. Así mismo, la presente descripción de actividades tiene como fin ser aplicada a estudiantes de $3^{\circ}$ de Educación Básica en aulas inclusivas.

En primer lugar, la adecuación del material fue enfocada en el cambio de texturas de cada una de las partes, pues como ya se mencionó el minicomputador cuenta con cuatro divisiones en cada una de sus placas y cierta cantidad de fichas que deben ser ubicadas en las partes divididas, por consiguiente la parte blanca, roja, rosada y marrón que representan dichas partes son cambiadas por fomi liso, fomi corrugado, tela y metal. En cuento a las fichas estas serán añadidas con velcro o con piezas de metal que se adhieran con la ayuda de un imán, esto con el fin de que las fichas permanezcan en un sitio fijo de la tabla.

Para las actividades propuestas, en cuanto al aprendizaje de las 4 operaciones básicas se espera:

Actividad 1: Reconocimiento del material de trabajo y reglas para su correcto uso.

Actividad 2: Construcción e identificación de números de base 10 y base 2 .

Actividad 3: Suma y resta de números de 1 y 2 cifras en las respectivas bases 10 y 2 .

Actividad 4: Multiplicación y división de números de 1 y 2 cifras en las respectivas bases 10 y 2.

No obstante el número de actividades puede variar si se quiere enseñar más operaciones numéricas y así mismo el grado de dificultad que se implemente ya que se podrían realizar actividades con más de 2 placas.

\section{Logros y dificultades evidenciadas.}

Dentro de este marco es importante considerar las hipótesis que se plantean ante la implementación de la propuesta de enseñanza.

- Los estudiantes con baja visión asimilan la adaptación del material y comienzan a desarrollar estrategias para poder operar.
- En el aula inclusiva el uso de material mejora las relaciones entre estudiantes videntes y de baja visión logrando una verdadera inclusión en donde se valoran las capacidades de quien nos rodea.

- Las operaciones básicas son asimiladas con el uso del material y esté potencia el razonamiento lógico en los estudiantes, además de llevarlos a encontrar otro tipo de aplicaciones como puede ser los números fraccionarios.

\section{Reflexión final}

Con la necesidad de dotar e implementar instrumentos eficientes que permita a estudiantes con limitación visual mejorar sus capacidades, tanto físicas como mentales y más aún en el campo de las matemáticas, el presente instrumento dará cuenta de aprendizaje tales como las operaciones básicas de la matemática, ya que estas son parte fundamental para el desarrollo de una persona. Es por esto que como estrategia lúdica este instrumento dará resultados sobresalientes ya que cuenta con las adaptaciones necesarias para que el estudiante no sea una persona excluida por su limitación en el proceso de enseñanza, sino que por el contrario pueda experimentar como los demás niños un proceso normal de aprendizaje.

Así mismo, los resultados obtenidos con este material didáctico podrán ser beneficiosos no solo para los estudiantes, quienes aprenderán la habilidad el manejo de algoritmos, sino también para los docentes, pues gracias a las adaptaciones realizadas y un buen conocimiento en el manejo del instrumento serán capaces de proporcionar a estudiantes con estas limitaciones buenas capacidades e impulsar la labor inclusiva que tiene la educación en nuestro país.

\section{Referencias}

Calderón, M., \& Vega, A. (2011). Elaboración de una guía del uso del material didáctico para el proceso de enseñanza aprendizaje en el área de la matemáticas para niños con discapacidad visual incluidos en el segundo año de educación básica. Cuenca - Ecuador: Universidad Politecnica Salesiana.

Robayna, M. S. (1989). El minicomputador de Papy: sugerencias didácticas. 49-61. 